resource is to apply a styptic to the interior of the uterus. But I see that a writer in the JOURNAL, in antagonising the use of perchloride of iron, says that, with one or the other plan, we can secure contraction, and therefore have no need of a styptic. I must say this remark fills me with astonishment; and $I$ think it must also others, quite as alive as I am, and as he is, to the importance of establishing reflex action and using all the remedies as sedulously as he recommends. Like Dr. Barnes, whose area of work embraced much of the same classes as my own, I hailed with a feeling of, I may say, extreme satisfaction, a plan which would give some hope of help, when every other attempt to establish uterine contraction had failed. To feel so powerless, when the patient was rapidly sinking before one's face ; to feel at the mercy of reflex function, and yet the last plan had failed to establish it, was an incubus in practice which I, with Dr. Barnes, and doubtless many others, once felt to be very disheartening. Of course, I do not mean to say that a great number of cases of hæmorrhage could not be arrested by the means already indicated ; but, I need scarcely argue for a moment, that some could not be checked, because I am sure everyone in large midwifery practice has seen such cases. And this is why I say that I hailed with great pleasure one more remedy in these desperate cases. Indeed, as soon as I had heard that Dr. Barnes was employing this remedy, which was shortly after he had begun to use it, I tried it ; and I merely give my own experience, when I say I have never seen an unfortunate result from its use; but $I$ have seen most marked effects in all cases in which it was employed in arresting hæmorrhage after other measures had failed. I may add to this, the confirmation of others with whom I have conversed, and I have not from them heard of a case fatal in consequence of it. It is true I have seen a woman already dying of hæmorrhage, not rally; but it would be absurd to attribute death to the remedy last used. But the writer referred to mentions all kinds of evils as having arisen from its use, and everything which occurs after is placed to its account. Puerperal fever, pyæmia, etc., are attributed to it. I can only say I know nothing of this in my practice. But surely it is not unusual for women after severe flooding (and this remedy is only used in the severest) to have septicrmia ; is it not well known, on the contrary, that they are very liable to it? Why, then, is the treatment to be credited with evils to which the condition for which the remedy was used gives rise ?

If, after all the controversy, one can only find record of some four or five deaths after, out of the great number of cases in which it has been used, one can only say that it is rather a proof that it prevented septicxmia than caused it.

It was a misfortune that the discussion on the subject at the Obstetrical Society last year was an indirect one. The paper which drew it forth was a description of a case of abortion in which solution of iron had been used, and in which it most probably was the cause of death. But, upon inquiry, the solution proved to be the strongest of the British Pharmacopoia. I think this was very much too strong; for the vagina cannot bear it without risk of damage, much less the uterus, more or less denuded as it is after abortion. It was at the least four times too strong, probably eight times, for any tissue like that of the uterus to bear, unless in a quantity so small that it could only cover it with a film. The discussion after this case was read turned, however, upon the iron treatment in post partum hæmorrhage; but, being aside the direct issue of the paper, it was not completely discussed, but nearly every one present spoke strongly in favour of the advantages of the iron treatment. At any rate, the case read was only a proof that the very strongest solution was fatal, which was the most probable result, and no proof of the danger of the weaker solutions, such as the tincture or weaker liquor of the British Pharmacopaia. In my own practice, I employ a rather weak solution at first-one of the weaker liquor ferri perchloridi to six or eight of water. If that fail, then I make it stronger; but, if the flooding be rapid, I use the full strength of the weaker solution or the tincture, because much of it is lost in the blood, or is prevented from coming easily into contact with the walls. The tube of the syringe should be carried to the fundus, and the fluid gently injected; or it may be conveyed with a sponge, which should be passed over all the internal surface. Perhaps this is the safest mode; but it must be removed promptly, or the uterus may retain it, as occurred in one case I know. I have tried it in all kinds of post partum hæmorrhage, after removal of clots, which is important, or they become coagulated and firm, and cannot be removed afterwards without trouble. I have found it particularly satisfactory in cases of irregular and intermittent relaxation, causing the uterus to assume its proper form at once.

I do not enter into the discussion as to how it acts. It stops blood in hæmorrhage from a wound on the outside; I presume it acts similarly on the inner side of the uterus-of course not requiring general uterine contraction. I have no doubt, in the less inert conditions, it rouses the remnants of contraction, and acts as cold and the presence of the hand. In conclusion, I will add that, till any one can show a better remedy, or till I find it of greater disadvantage than extreme loss or death, I shall continue to use it in the fullest confidence, or till it has been clearly pointed out that it is dangerous to use. For the proof of this, I have failed to find sufficient at present. In the only case given by the writer in the JOURNAL, where death seemed to be due to the injection, there was no post mortem examination, and therefore only presumptive evidence. The others have not been published. It would have been much more satisfactory to me, and I think to the profession generally, had Dr. Snow Beck given the other cases he says he had seen, without occupying so much space in personal remarks, which hinder much our arriving at the solution of the question, Is the iron treatment in post partum hæmorrhage dangerous? and, if so, is it more so than the condition for which it is used? If this treatment be safe, thanks, not abuse, should be given to the promoter. If not, let us scientifically discuss whether the danger can be eliminated. If it cannot, then let us put it aside, with regret that flooding is still a fatal condition, but nevertheless thanking the promoter for the effort to save human life, even though unsuccessful. I can only reiterate that I have freely used it up to the strength of the tincture without an untoward result. I have known cases where it has been used without calamity; and I have made numerous enquiries among medical gentlemen, and have failed to find a fatal case following its use.

With regard to the second subject-namely, what can be done to prevent hæmorrhage in "flooders"-of course the answer is, be always as prepared as one should be in an ordinary case; but I think it would be well to give secale very early in labour, in small doses, such as ten grains every two or three hours for four or six doses; or to rupture the membranes as early as possible in labour, or even before labour sets in.

I may add one more remark; that, although I do not despise the assistance, when single-handed, of spring-pads, etc., yet I consider that nothing equals the hand, which should never leave the uterus from the actual expulsion of the foetus till the uterus has continued firm for an hour after labour, or after cessation of hæmorrhage, or even longer after very dangerous flooding.

\section{THE ANTICIPATION OF POST PARTUM HÆMORRHAGE.}

By G. T. GREAM, M.D., F.R.C.P., etc.

IN alluding to the remarks on the "Anticipation and Treatment of post partum Hæmorrhage," contained in the very interesting communications on the subject which have appeared in the JOURNAL, and which are deserving of great consideration, as showing much practical experience and a full appreciation of the difficulties which have to be overcome, it is not my intention, in any one particular, to differ from the views of others as to the extreme danger attending uterine hæmorrhage; for is there any responsibility so great in any department of the medical profession as attending cases of the kind ? and yet how lightly do some people estimate the responsibilities of the practice of midwifery. There are those who say that midwifery can be attended by any one; and the Obstetrical Society itself goes so far as to solicit Government aid in en. forcing the education of midwifes, and proposes to undertake an examination of such women, and to grant them diplomas.

I know that arguments are used to explain away some of the objectionable parts of these proceedings, but the very contemplation of them is derogatory alike to the profession, to science, and to the Cbstetrical Society. Without, therefore, calling in question the views of the writers in the JOURNAL, as to the great importance of the subject, I vet cannot help thinking that the treatment proposed by some of them is a little more extended than is wanted; and, without pointing to any one correspondent in particular, a feeling arises that most of them urge local measures too much, instead of more general remedies, and they run somewhat in the groove too apparent in the writings of recent authors on uterine diseases, who ignore very nearly all medicinal remedies, and resort to local, and, in my idea, inefficient and often hurtful agents. No fewer than three, if not more, uterine "stems" as they are called, were exhibited at the last meeting of the Obstetrical Society by their respective inventors. Are such things ever necessary? and are they not productive of immediate or of deferred disease? Two fatal cases of local interference purporting to remove sterility in healthy women came before me in the same week, and the extent of the injury inflicted on women by locally treating them, can only be known to those who have the opportunity of personally witnessing it: 
Without imagining that my opinions will be preferred to those of other writers, I yet think that a rather extensive experience may be my apology for suggesting that, instead of injecting iron, iced water, or chloralum, placing the child to the breasts, compressing the aorta, using galvanism, special " binders," or transfusion (to prepare or employ either of which mode of treatment involves necessarily more than the few minutes required for the death of the patient in such cases), the use of remedies sure to be at hand should be preferred, of which brandy, in my view, is the chief.

It was taught by an eminent physician many years ago in his lectures, that, if death resulted from hæmorrhage, while a supply of brandy was at hand, the fault was attributable to the medical attendant : a bold assertion to make, no doubt, but nevertheless a true one. In such cases all the remedies wanted are brandy and ergot, and in addition, if possible, ice. The administration of brandy is too lightly thought of -it is mentioned by some recent writers in the JOURNAL, but not as the chief remedy. It may be given to any extent required, the pulse alone being the indi. cator as to quantity, mixed with equal parts of water, and poured down at short intervals, should the flagging heart require it. The stomach will reject it, almost as a rule, the moment vitality is restored; and if it be retained, which it sometimes but very rarely is, no harm will come of it. If the left hand grasp the uterus through the abdomen so firmly that it cannot dilate, while the right removes the placenta, as well as all coagula that may form in the uterus, and, if there be ice, carries portions of it within the cavity, no other remedies will be wanted. As vitality is restored, the uterus contracts, and all danger is over: then is the time to give nourishment. Free air should be admitted by open windows, and from the moment uterine contraction is established, the warmth of the body should be promoted.

It has always seemed to me that the use of abdominal bandages is but little understood as a remedy ; no bandage can be so firmly placed as to prevent the dilatation of the uterus, while the hand firmly fixed on it can grasp it with such power that dilatation and relaxation are impossible; and, until contraction has been complete for at least half-an-hour, the hand should not be removed. By the hand is learnt the condition of the uterus also; and the occurrence of what is called secondary hæmorrhage is entirely due to too much faith being placed in bandages, under which the uterus will be filling with blood unobserved, until the occurrence of alarming syncope.

The injection of perchloride of iron is not likely to become a popular remedy. It is known to produce mischief, which I myself have witnessed, and even death; and besides, it is never required. I have also witnessed the fatal effects of injection of iced water, as well as its inefficiency in checking uterine hæmorrhage. It was injected by a very able medical man before my arrival, in a case to which $\mathbf{I}$ was called; the patient complained of intense coldness over the whole abdomen, accompanied by pain. She was not faint, but she died as those die who have received peritoneal injury. It was the opinion of both the medical attendant and myself, that solid ice introduced into the uterus was preferable to iced water injection, for we were both impressed with the conviction that the iced water had here reached the peritoneum through the Fallopian tubes.

To prevent post partum hæmorrhage, does not require quite the same energy as to control it when it occurs. Time is not so pressing. When women have once flooded, they are liable to do so again; the general health of such women should be attended to during pregnancy; tone should be given to the abdominal muscles especially; the remedies, however, must be adapted to the patient's condition. When labour begins, the pulse should be well sustained by nourishment and stimulants, brandy in fair quantities being given. The use of ergot as a preventative is most important; the writers in the JOURNAL allude to its use, but they differ as to the dose, and as to the time of giving it. The effect of ergot is certainly not apparent under twenty minutes; hence I am not disposed to think it useful when given at the moment of the expulsion of the foetus. As nearly as possible, it should be given twenty minutes, therefore, before the birth, and I have never found the effect produced by any less dose than sixty minims. It never injures the child when administered judiciously ; but the dose should be, under any circumstances, not less than sixty minims of the fluid extract of the Pharmacopocia.

The labour should not be hurried, and it is as well to avoid giving ergot during its progress, except the single dose mentioned. As the foetus is expelled, the left hand, as suggested by more than one of the recent writers, should firmly press the uterus downwards, following it so that it still receives the pressure, not only after the birth is accomplished, but until after the placenta has also been expelled. No doub the presence of the placenta, whether wholly or partially detached, excites bleeding; hence there should be no great delay in its being removed, but no forcible extraction should be adopted. My own expe- rience goes to prove that this mode of treatment will always be successful, and that there will be no need, if it is adopted, of the anticipation of the necessity for transfusion as suggested by one of the correspondents of the JOURNAL.

The is still another form of hremorrhage not noticed in these com muinications. I allude to bleeding during pregnancy, and especially after the seventh month, or at the fall term of gestation, before labour has commenced. There is no condition requiring more judicious management than this contingency at the latter months, and none so likely to be fatal unless properly treated. Perhaps others, who have not written, will record their views on this subject on some future occasion; and, if this be done with the same ability with which they have written upon the other forms of hæmorrhage, their suggestions will be productive of the greatest good.

\section{THE DEPRESSANTS OF THE CIRCULATION AND} THEIR USE.*

\section{By J. MILNER FOTHERGILL, M.D., M.R.C.P.,}

[Concluded from p. 47 of last number.]

NITRITE of amyl is an agent which produces, when inhaled, a distinct and remarkable blushing, obviously from its dilating the minute peripheral vessels. Some years ago, Dr. Lauder Brunton found that, in a case of angina pectoris which came under his notice, there was a decided increase of arterial tension in the attack ; and this he attributed to peripheral spasm. He reasoned, and rightly too, that nitrite of amyl ongit to relieve this condition, and the practical outcome of this application of physiological knowledge was the relief of the patient. A more perfect demonstration of the power of physiological research to aid practical medicine is not necessary to disprove a charge often pressed. More recently, Dr. Crichton Browne used amyl as a test of the vasomotor mobility of epileptics, and has found them extremely susceptible to its action. He tried it in the treatment of the fit, however, without success. Observing that there is a rise in the arterial tension at the instant of the oncome of the fit, he tried inhalations of the amyl just before the oncome of rhythmically recurring fits with the following result.

E. W., aged 27, had, from January Ist to March 26th (eighty-five days), no less than eighty fits, usually daily, and during March, one each day. On March 27th, amyl inhalations were commenced. The fits were immediately and abruptly discontinued; and there was no recurrence of them till April 15 th. The fits have returned, but unfrequently, though the inhalations had been almost abandoned (West Riding Lunatic Asylum Reports, vol. iii).

In Dr. Ferrier's experiments as to the localisation of the functions of the brain, commenced at Wakefield Asylum last Easter, it was very pretty, as well as instructive, to see how the inhalation of amyl previously to the application of the electrodes to the entire cerebral hemisphere on one side, arrested the unilateral fit which would have been, and was otherwise, produced.

Further experience of nitrite of amyl at Wakefield is accumulating testimony as to its power over recurrent epilepsy, and in averting that miserable condition which is the consequence of recurrent fits, called the status epilepticus.

There seems every reason to believe, though as yet it is not possible to be more certain, that in inhalations of nitrite of amyl will be found the most efficient, as well as agreeable method of treating attacks of hysteria, where there is spasm of the arterioles.

Nitrite of amyl will probably also be found useful in the treatment of chronic Bright's disease when the tension is high, the pulse corded, the action of the heart violent, often amounting to palpitation, the flow of limpid urine profuse, and the sleeplessness and mental irritability pronounced. Dr. Balthazar Foster is kindly testing the amyl in these conditions for me; but his results have not yet come to hand. One interesting case, illustrating well the action of amyl, he has already furnished to me. He says: "I used it in some heart-cases. In one especially it did well-a case of aortic insufficiency-with great hypertrophic compensation. In this case, there were great frontal headache and very violent heart-action, associated with high arterial tension. The nitrite of amyl relieved these conditions like magic."

Dr. Brunton has kindly placed at my disposal three sphygmographic tracings of the pulse in angina before and after the inhalation of amyl. The first figure gives the pulse in its normal condition, as seen before the attack came on, and after the attack had entirely passed off. (Fig.

* Read before the Medical Society of London. 